\title{
Medium-sized tandem repeats represent an abundant component of the Drosophila virilis genome
}

\author{
Murat A Abdurashitov', Danila A Gonchar', Valery A Chernukhin', Victor N Tomilov', Julia E Tomilova', \\ Natalia G Schostak², Olga G Zatsepina ${ }^{2}$, Elena S Zelentsova ${ }^{2}$, Michael B Evgen'ev' ${ }^{2,3^{*}}$ and Sergey Kh Degtyarev ${ }^{1}$
}

\begin{abstract}
Background: Previously, we developed a simple method for carrying out a restriction enzyme analysis of eukaryotic DNA in silico, based on the known DNA sequences of the genomes. This method allows the user to calculate lengths of all DNA fragments that are formed after a whole genome is digested at the theoretical recognition sites of a given restriction enzyme. A comparison of the observed peaks in distribution diagrams with the results from DNA cleavage using several restriction enzymes performed in vitro have shown good correspondence between the theoretical and experimental data in several cases. Here, we applied this approach to the annotated genome of Drosophila virilis which is extremely rich in various repeats.

Results: Here we explored the combined approach to perform the restriction analysis of D. virilis DNA. This approach enabled to reveal three abundant medium-sized tandem repeats within the $D$. virilis genome. While the $225 \mathrm{bp}$ repeats were revealed previously in intergenic non-transcribed spacers between ribosomal genes of D. virilis, two other families comprised of $154 \mathrm{bp}$ and $172 \mathrm{bp}$ repeats were not described. Tandem Repeats Finder search demonstrated that $154 \mathrm{bp}$ and $172 \mathrm{bp}$ units are organized in multiple clusters in the genome of $D$. virilis. Characteristically, only 154 bp repeats derived from Helitron transposon are transcribed.

Conclusion: Using in silico digestion in combination with conventional restriction analysis and sequencing of repeated DNA fragments enabled us to isolate and characterize three highly abundant families of medium-sized repeats present in the $D$. virilis genome. These repeats comprise a significant portion of the genome and may have important roles in genome function and structural integrity. Therefore, we demonstrated an approach which makes possible to investigate in detail the gross arrangement and expression of medium-sized repeats basing on sequencing data even in the case of incompletely assembled and/or annotated genomes.
\end{abstract}

Keywords: Drosophila virilis, in silico digestion, Medium size repeats, Evolution

\section{Background}

Though multiple plant and animal genomes have been sequenced and annotated, including many from Drosophila, abundant fractions of repeated DNA often forming heterochromatic regions of the genome escape description. Large heterochromatic segments of genomes remain poorly analysed because the repetitive nature of the DNA present in heterochromatin makes cloning, assembly and

\footnotetext{
* Correspondence: misha672011@yahoo.com

2Engelhardt Institute of Molecular Biology, Vavilov str. 32, Moscow 119991, Russia

${ }^{3}$ Institute of Cell Biophysics, Pushchino 142290, Russia

Full list of author information is available at the end of the article
}

annotation very difficult. Heterochromatic regions are the dark matter in genomes and even for well studied organisms we still do not have a complete genomic sequence due to the difficulties of sequencing these regions. Previously, we developed a simple method for carrying out a restriction enzyme analysis of eukaryotic DNA in silico, based on the known DNA sequences of the genomes [1]. This method allows the user to calculate lengths of all DNA fragments that are formed after a whole genome is digested at the theoretical recognition sites of a given restriction enzyme. The program also constructs distribution diagrams of the calculated restriction DNA

\section{Biomed Central}

(C) 2013 Abdurashitov et al.; licensee BioMed Central Ltd. This is an open access article distributed under the terms of the Creative Commons Attribution License (http://creativecommons.org/licenses/by/2.0), which permits unrestricted use, distribution, and reproduction in any medium, provided the original work is properly cited. 
fragments. These distribution diagrams display distinct peaks, where DNA fragments of definite lengths are present due to DNA repeats in eukaryotic genomes. A comparison of the observed peaks in distribution diagrams with the results from rat, mouse and human DNA cleavage using several restriction enzymes performed in vitro have shown good correspondence between the theoretical and experimental data [2,3]. Here, we applied this approach to the annotated genome of Drosophila virilis.

Satellite and minisatellite DNAs constitute a considerable part of the genomic DNA and are often found as runs of thousands or more copies of unit sequences (100-300 bp and 3-15 bp, respectively) predominantly localized in heterochromatic regions. SatDNA is generally formed by long tandem arrays in which the units are repeated in a head-to-tail fashion [4,5]. More than 40\% of the $D$. virilis genome consists of three simple minisatellite DNAs, each of which are seven base pairs long, that are located predominantly in pericentromeric heterochromatin in all chromosomes of species within the virilis phylad [6].

D. virilis represents the most karyotipically primitive species of the virilis phylad [7,8]. The availability of a sequenced genome enables the application of our in silico digestion method to look for the presence and abundance of repeats that were not adequately described in the sequenced genome of this species, due to limitations of current sequencing and mapping techniques for assembling tandem repeat motifs scattered throughout the genome.
In past decades, an extensive analysis of various classes of repeats, including cryptic satellites and various classes of mobile elements in $D$. virilis and other species of the virilis group, have been performed in our laboratory and by other groups [9-14]. Therefore, it was of significant interest to extend our analysis to the repeated fraction of the $D$. virilis genome and to explore in silico digestion in combination with conventional restriction analysis. These analyses help to reveal and describe uncharacterized and highly abundant families of repeats within the genome of this unique in many ways [6,7], species of Drosophila. Here, we describe three highly abundant families of medium-sized tandem repeats within the $D$. virilis genome. The consensus repeat units comprising these families were cloned, sequenced and compared with those of the related species $D$. americana. This analysis emphasizes the validity and versatility of the in silico digestion method in studying various repeats often included in the heterochromatic fraction of genome.

\section{Results and discussion}

Several families of medium-sized tandem repeats are revealed by in vitro and in silico restriction analysis in $D$. virilis genomic DNA

We performed hydrolysis of $D$. virilis genomic DNA with different restriction endonucleases. Figure 1 shows the patterns of $D$. virilis DNA cleavage with 12 restriction endonucleases. According to the data presented in Figure 1, DNA hydrolysis with restriction enzymes results in the formation of a few distinct visible bands.

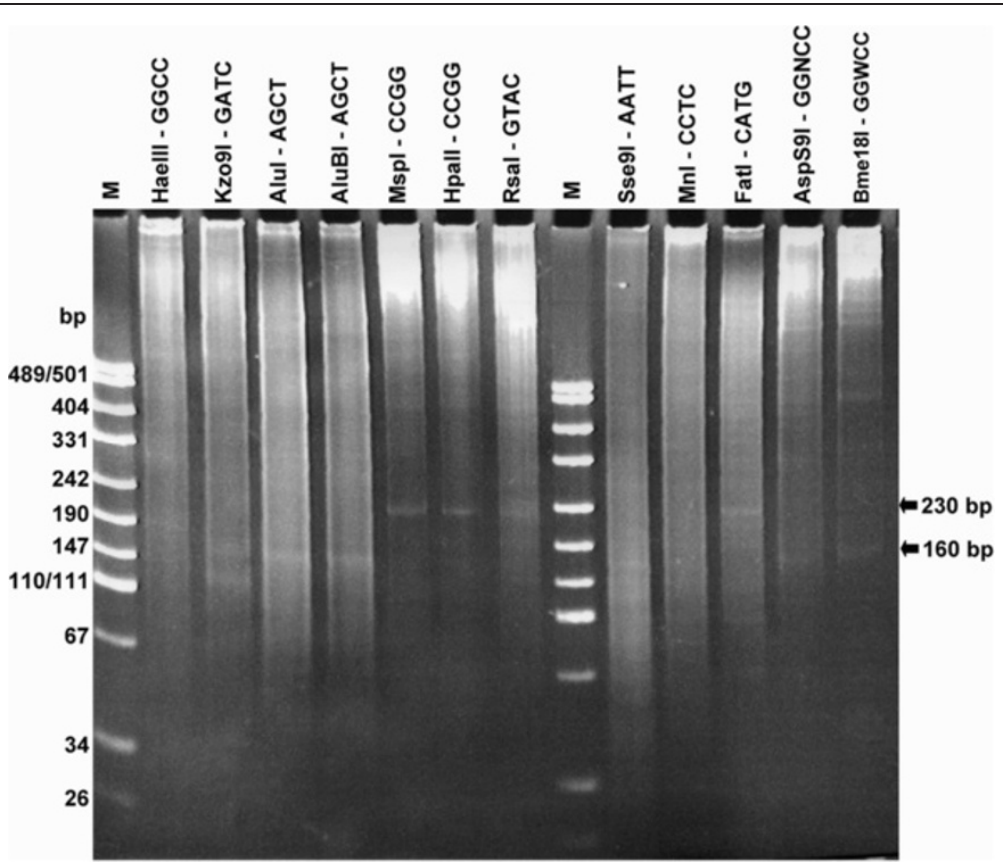

Figure 1 Electrophoretic separation of $D$. virilis DNA fragments produced by restriction enzyme hydrolysis. 
Interestingly, some of the restriction endonucleases produce DNA fragments of the same length. For example, a DNA fragment of $\sim 230 \mathrm{bp}$ is observed after DNA hydrolysis with MspI/HpaII, RsaI and FatI, whereas 160 bp DNA fragment is clearly observed after digestion with Kzo9I, AluI/AluBI, AspS9I and Bme18I (Figure 1).

The results of our study as well as genome sequencing data show that several families of abundant repetitive elements of medium size exist in the $D$. virilis genome. Moreover, the presence of same-size fragments in patterns of DNA cleavage with different restriction enzymes is a clear indication that these repetitive sequences are arranged in tandem. Therefore, based on the experimental data depicted in Figure 1, the tandem repeats of approximately $160 \mathrm{bp}$ and $230 \mathrm{bp}$ in length are present in the $D$. virilis genome in high copy number.

Satellite DNAs, which form heterochromatin regions in eukaryotic genomes, are the major source of tandem repeats in most of the genomes studied. However, Drosophila satellite DNA with a few prominent exceptions, includes very short repetitive sequences of 4-14 bp in length, depending on the species $[4,9,15]$.

We analyzed a known structure of the $D$. virilis genome to find medium-sized tandem repeat candidates. We performed in silico DNA digestion of the currently available draft $D$. virilis genome sequence, using recognition sites for the restriction endonucleases AluI, Kzo9I and HpaII, according to the earlier published protocols [1]. These restriction enzymes recognition sequences were chosen because they produce clearly visible DNA fragments.

Figure 2 summarizes the distribution of the fragment lengths obtained in the in silico digestion. According to the distribution, DNA hydrolysis with HpaII results in the formation of a DNA fragment of 225 bp in length, AluI hydrolysis produces a 154 bp DNA fragment and Kzo9I digestion gives three distinct DNA fragments that are 36, 118 and $154 \mathrm{bp}$ in length. These data correspond to the experimental results presented in Figure 1, except the 36 bp fragment.

It is noteworthy that DNA fragments that are shorter than $100 \mathrm{bp}$ are not usually observed on the gel (Figure 1) because their combined molecular mass remains below detection level [1].

Independently, we scanned the $D$. virilis genome using the Tandem Repeat Finder software to find tandemly arranged repetitive elements that were $40-500$ bp in length (Figure 3).

A comparison of the diagrams in Figures 1, 2 and 3 shows consistent results achieved by the three independent approaches, except the presence of an additional DNA peak that is $172 \mathrm{bp}$ in length and clearly observed in Figure 3.

Therefore, according to our results, there are multiple tandem repeats in the $D$. virilis genome that are much longer than the previously described minisatellite sequences, and they are unrelated to the pvB370 satellite family and pDv family described in this species $[9,15]$. The origin and genomic location of 154, 172 and $225 \mathrm{bp}$ fragments that comprise significant parts of the $D$. virilis genome are discussed below.

\section{5 bp tandem repeats represent intergenic spacers between ribosomal genes}

The ribosomal DNA (rDNA) of insects contains several hundred structural-functional units arranged in tandemly repeated clusters in nucleolus organisers, separated by several transcribed and nontranscribed spacers. Tandem repeats of $225 \mathrm{bp}$ in DNA of $D$. virilis have been noted elsewhere [16]. These 225 bp repeats are located in IGS (intergenic spacer) between $28 \mathrm{~S}$ and $18 \mathrm{~S}$ rRNA genes. It was suggested that, in Drosophila, these repeats are not transcribed and most likely serve as enhancers of gene expression [17]. Ribosomal RNA genes in Drosophila form clusters that are abundant (i.e., several hundreds of copies) within the genome. Each Drosophila species contains tandem repeats of defined length within an IGS region [18]. Figure 4 shows a consensus DNA sequence of a $D$. virilis IGS tandem repeat (225 bp), with highlighted recognition sites of restriction endonucleases RsaI, FatI and HpaII/MspI.

There are unique sites for RsaI and HpaII/MspI within the IGS tandem repeat, and its cleavage with the indicated restriction enzymes should result in the formation of 225 bp DNA fragments and thus correspond to the experimentally observed data (Figure 1). Surprisingly, the consensus IGS tandem repeat contains two sites recognized by the FatI restriction enzyme.

To confirm the origin of the visible fragments, we have purified the 225 bp HpaII fragments from the gel for cloning and sequencing. Eight of the twenty-eight obtained sequences exhibit a high degree of similarity (96-99\% identity) to the $225 \mathrm{bp}$ consensus sequence (Figure 5) while the remaining twenty sequences exhibit no significant homology to the consensus (data not shown).

According to Figure 5, two of the eight sequenced HpaII fragments carry a mutation in the first Fat I recognition site, which may explain the presence of a $225 \mathrm{bp}$ DNA fragment in the hydrolysis products from this enzyme.

We performed an in situ hybridization of a 225 bp HpaII probe (plasmid $\mathrm{pHpaV}-\mathrm{kl} 22$ ) with $D$. virilis salivary gland polytene chromosomes; as expected, we observed significant hybridization in the heterochromatic chromocenter and multiple diffuse grains in a restricted region of the nucleolus (Figure 6A). Our Northern hybridization experiments using total RNA and labelled 225 probe demonstrated that these repeats are most likely not transcribed in $D$. virilis because we did not observe any transcription in the $D$. virilis strain 


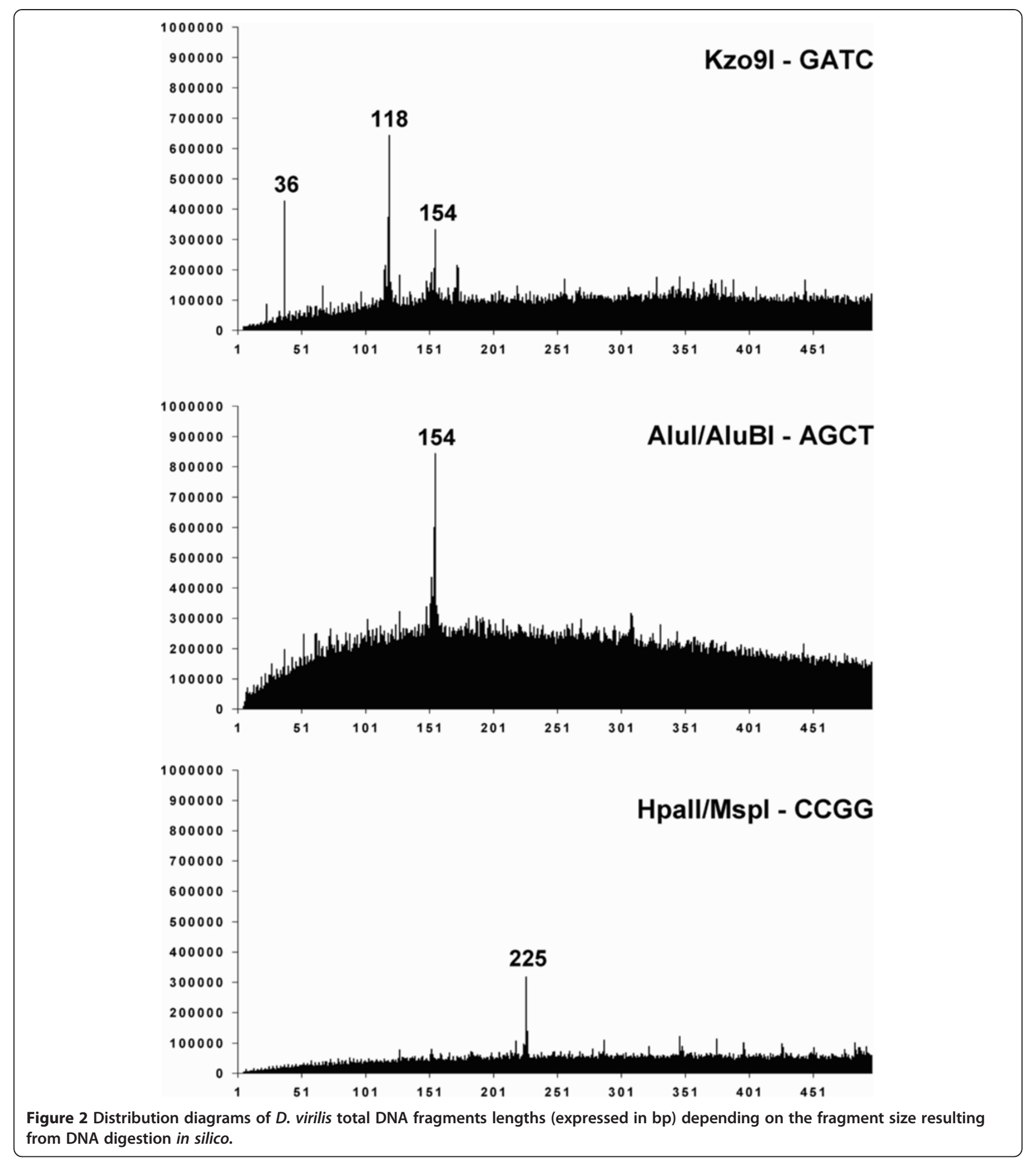

used for analysis (strain 160) of the tandem repeat and only weak transcription in another $D$. virilis strain (strain 9), which probably represents a read-through transcription of this repeat. Furthermore, we failed to observe any hybridization with poly (A)-RNA of both D. virilis strains in Northern blots using the labeled 225 probe (data not shown).
The 154 bp tandem repeat family is apparently derived from Helitron transposable element

Tandem repeats of $154 \mathrm{bp}$, as indicated in Figures 1, 2 and 3 , are not described in the literature to our knowledge. However, results from the Tandem Repeat Finder show that there are 2219 clusters in the genome that contain 153-154 bp tandem repeats. There are approximately 


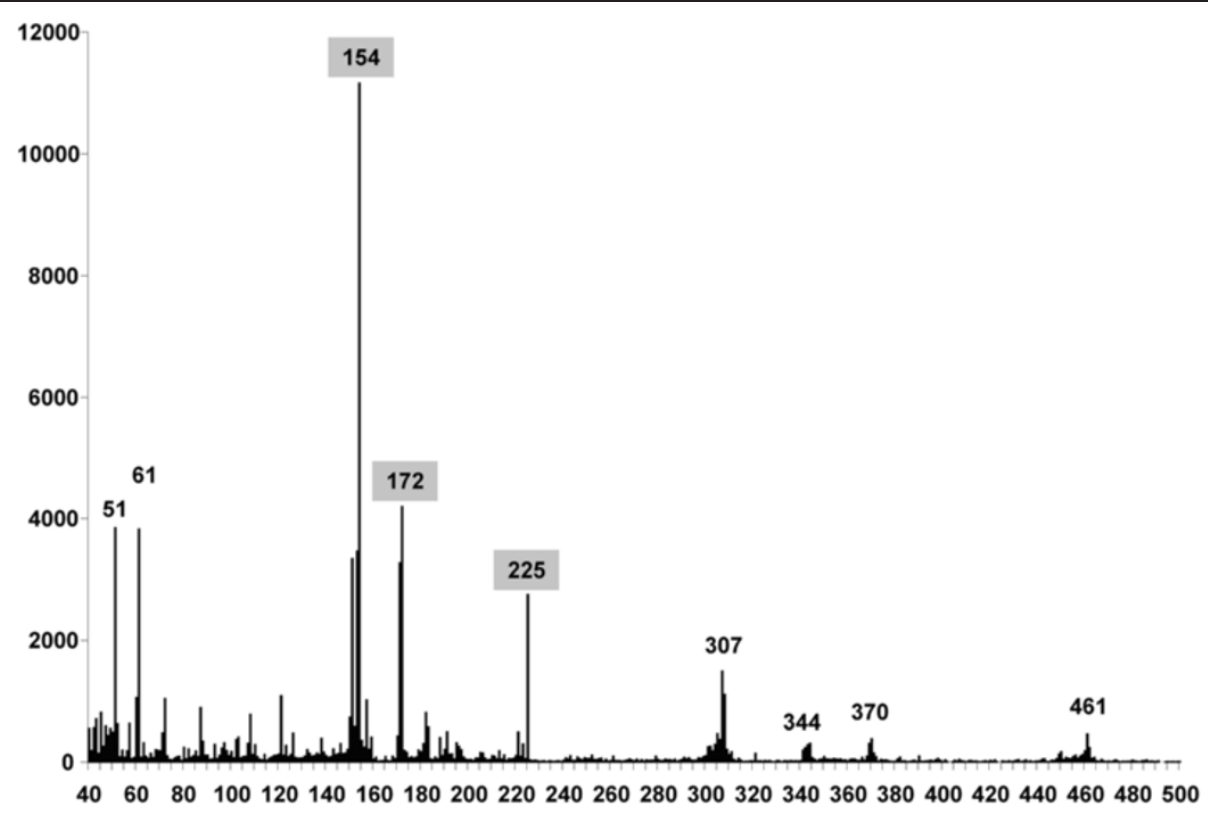

Figure 3 The quantity of tandemly arranged repeats of $\mathbf{4 0 - 5 0 0 ~ b p ~ l e n g t h ~ i n ~ t h e ~} D$. virilis genome. Abscissa axis - length of fragment in bp; ordinate axis - number of copies. The major peaks are indicated above the diagram.

14160 individual repeat units of this length. To determine the origin of the 153-154 bp repeat, we have extracted all consensus sequences from the table produced by the Tandem Repeat Finder and assemble them in one consensus sequence. It is of note, that $118 \mathrm{bp}$ and $36 \mathrm{bp}$ fragments in Kzo9I distribution diagram apparently appear due to hydrolysis of $154 \mathrm{bp}$ fragment and further analysis (see Figure 7) confirms this assumption.

The comparison of the consensus sequence with the REPBASE database $[19,20]$ shows that the 153-154 bp fragment is derived from the Helitron-2 interspersed repetitive element. The full length of the intact Helitron-2 transposon of D. virilis is $9141 \mathrm{bp}$, and the 153-154 bp consensus sequence exhibits a high degree of homology to the region found between positions 237 and 1087. This particular region contains four copies of the 153154 bp repeat within the full length of the consensus sequence of Helitron-2. A map of this Helitron-2 fragment is depicted in Figure 7.

This consensus sequence contains two GATC sites (i.e., Kzo9I recognition sites) in each unit, but we still can see the presence of intact $154 \mathrm{bp}$ fragments in Figure 2, which means that many 154 bp fragments include only one Kzo9I recognition site.

Full-length Helitron-2 elements are not abundant in the $D$. virilis genome, but there are a lot of truncated copies that mainly include the first 928 bp fragment 5' of the transposon. In general, Helitron-2 fragments of different length occupy as much as $\sim 5 \%$ of $D$. virilis genome [21]. Thus, the 153-154 bp DNA fragments that are visible in the gel (Figure 1) may be explained by the presence of multiple, predominantly truncated, copies of this transposon representing the remnants of the Helitron amplification process that occurred at some point in the virilis group evolution.

It is noteworthy that abundant DINE-I transposable element has been described in 12 species of Drosophila, including $D$. virilis. It was proposed that DINE-1 is also related to Helitrons, a family of DNA-mediated transposons [22]. However, our analysis demonstrates that the 154 bp tandem repeats are definitely not included in DINE-I transposon sequences in D. virilis.

\section{TAGTACATGAAGACATTAAGGTGAATGGTAGCATTTGGAAAAAATATCGCCATTATAGAT RsaI (GTAC) FatI (CATG) \\ 61 GATGTGTCAAAAAACCTATATAGGGAGTGGTGTCGCGCAAGGCATGTCATATGAAAAATT Fat I (CATG) \\ 121 TTAAATACGGTAAATTCATATGACTGTGGAGTGTTGCCTTGCCGGCATAAGTCAATTATG HPaII (CCGG)}

181 TTTATATAAGGAGAATAATGAAGTTATAAAAGTGTATTATTAAAT

Figure 4 Recognition sites of three restriction enzymes in the consensus sequence of the $225 \mathrm{bp}$ tandem repeat. 
To describe the distribution of 154 bp family of repeats in the chromosomes of $D$. virilis we carried out in situ hybridization of salivary gland polytene chromosomes with a 350 bp probe that was obtained by PCR from $D$. virilis DNA and included two 154 bp repeat units. As expected, the experiments revealed a very strong hybridization in the chromocenter and multiple sites of hybridization scattered in the chromosomes (Figure 6B). A Northern blot analysis demonstrated that the $154 \mathrm{bp}$ repeats are present in the poly (A) (+) fraction of RNA because the correspondent probe hybridized with a high molecular weight $(10 \mathrm{~kb})$ band in both of the $D$. virilis strains studied, but not in D. melanogaster (Figure 8). Although the size of the hybridization fragment corresponds to the full-size transcript of Helitron-2 (approximately $9 \mathrm{~kb}$ ), it will be necessary to use other probes complementary to this transposable element to prove that Helitron is really transcribed in $D$. virilis species.

Multiple 172 bp tandem repeats are located in the ap gene of $D$. virilis and most likely in many other sites of the genome Surprisingly, we do not observe the $172 \mathrm{bp}$ fragment in the experimental digestion (Figure 1), and by in silico restriction analysis (Figure 2) although an investigation of the sequenced $D$. virilis genome using the Tandem Repeat Finder revealed a high peak at this fragment length (Figure 3). It is noteworthy that there is similarity in the monomer length of many centromeric satellites (often approximately $170 \mathrm{bp}$ ), which leads to the assumption that such a repeat unit might reflect uniformity in nucleosome phasing and heterochromatin propagation [5]. However, we failed to find any family consisting of sequences of this length in any studied Drosophila genomes with the exception of $D$. ananassae [24].

According to our analysis the number of 171-172 bp repeats in the sequenced $D$. virilis genome is 7455 and the number of genomic clusters that contain such units is 778 . We aligned most of the isolated $171-172$ bp sequences and obtained the following consensus sequence:

TACCATSAAATATCCTACATAGACATAGGTCGAAA ATTCCCAACCCCATAACTCGGCCAAAACTCAACCGA TTTTCATAAGGTWTAMMTTTTTGTTCATGGTTTGA CCTCWATATCAATCTGGCATATAAATCTGACAACTT TATTTTTGGTCAAAATTCATGTGAAAATGG.

The BLAST search for homologous sequences was performed and revealed one region of the $D$. virilis 


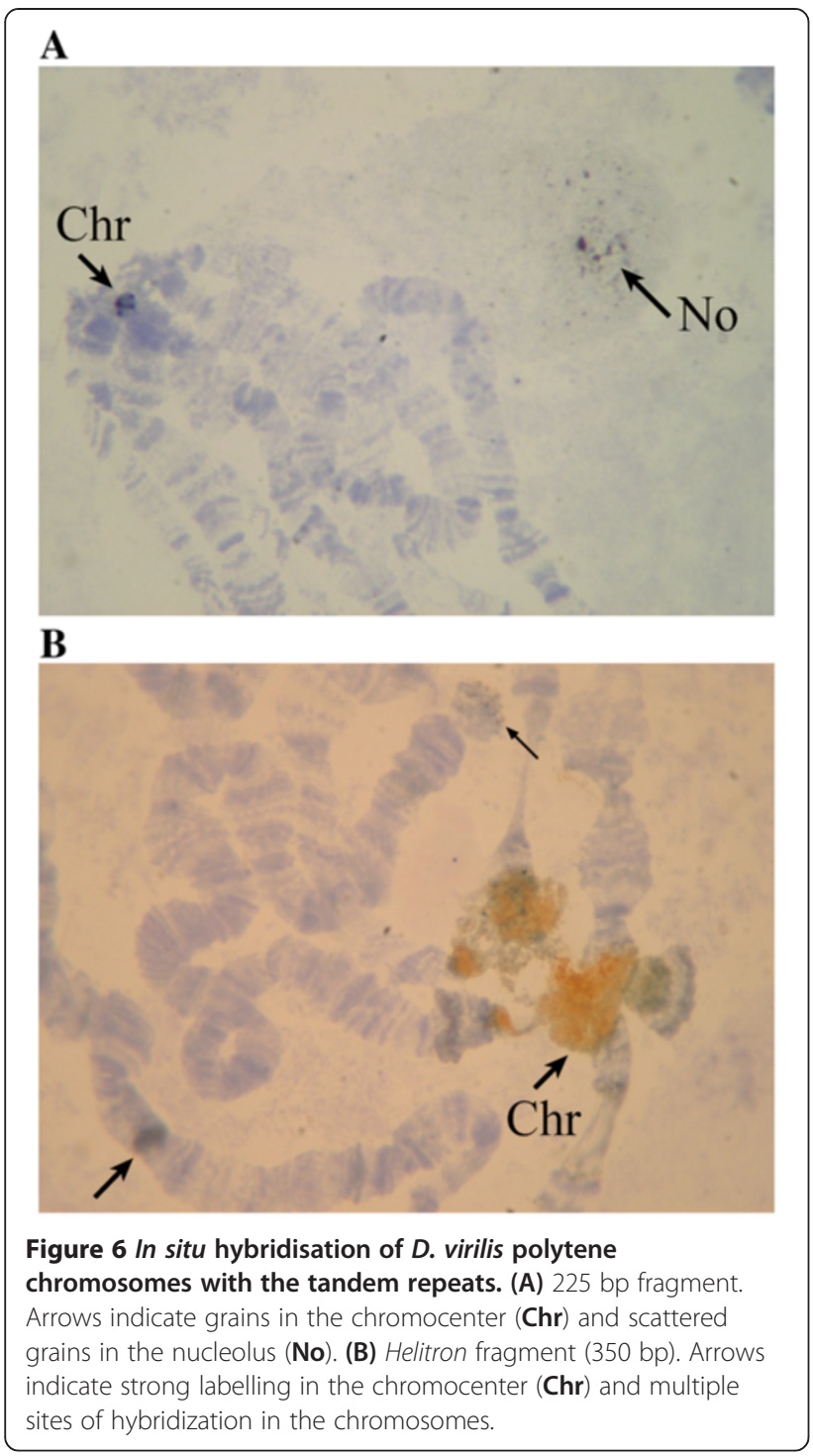

genome which contains multiple copies similar to the consensus sequence.

The region is located before the apterous (ap) gene of $D$. virilis (GenBank acc.no. AY186999). In D. melanogaster, this gene contains a homeodomain and encodes a key developmental regulatory protein [25]. In $D$. virilis, this genetic region contains 29 tandem units 172 bp in length, as well as other homologous sequences of different lengths. The general organization of this region in the latter species is depicted in Figure 9.

The structure and sequences of individual repeats included in the apterous cluster and their alignment are summarised in Additional file 1: Figure S1. The analysis indicates that the cluster of $172 \mathrm{bp}$ tandems located near the apterous gene has a rather complex structure. Blocks of 172 bp repeats (2-6 units) are interrupted by sequences of $161 \mathrm{bp}$ and $28-29 \mathrm{bp}$ in length, which represent the fragments of the same basic $172 \mathrm{bp}$ consensus sequence. Short 28-29 bp fragments always end with a hexanucleotide motif that is not homologous to the consensus sequence; after this motif, the hexanucleotide $161 \mathrm{bp}$ fragment lacking $11 \mathrm{bp}$ at the 5 ' end is always observed. All 172 bp units contained in the cluster exhibited amazingly high levels of identity (Additional file 1 : Figure S1), which suggests the concerted evolution of the sequences. Furthermore, the whole cluster, except for 29 full-size 172 bp units, contains $125^{\prime}$-deleted copies of the consensus sequence, 13 fragments $28-29 \mathrm{bp}$ in length and single fragments that are 170,173 and $175 \mathrm{bp}$ in length.

According to our Tandem Repeat Finder analysis, there are other clusters comprised of homologous 171-172 bp tandem repeats in the $D$. virilis genome, but the absence of a well annotated genome prevents the determination of their locations. We do not yet know whether the described $172 \mathrm{bp}$ cluster has something to do with apterous function, and we did not find any relevant information in the literature [26]. The role of this family of tandemly arranged sequences may also include regulation of gene activity, as in the case of the $225 \mathrm{bp}$ tandem repeats.

Unlike the $154 \mathrm{bp}$ and $225 \mathrm{bp}$ tandem repeats, DNA fragments of 171-172 bp in length isolated from different genomic regions (data not shown) display a high level of variability in the sequence. This difference may explain why the band that corresponds to the 171$172 \mathrm{bp}$ fragment was not present in the experimental (Figure 1) and in silico digestion (Figure 2).

\section{Investigation of the three major tandem repeats families in the genome \\ D. americana, another species of the virilis group}

$D$. americana belongs to the virilis phylad of the virilis group and is separated from $D$. virilis by $4-5$ million years of divergent evolution $[7,8,14]$. Given the evolutionary relationship between $D$. virilis and $D$. americana we were interested in comparing the abundancy of the medium-sized repeats within the genomes of these two species. The $D$. virilis which basing on most primitive karyotype lacking intraspecific rearrangements, maximal content of satellite DNA among species of the group and many other features appears to be more primitive of the two and may have features in common with the ancestral species of the whole virilis group $[7,8,14]$. Fortunately, the genome of $D$. americana is now completely annotated [27] and it is possible to perform BLAST searches for sequences of interest. We used this option to look for the presence of the $225 \mathrm{bp}, 154 \mathrm{bp}$ and 172 bp consensus sequences, which were previously detected in the $D$. virilis genome, in the annotated $D$. americana genomic sequences.

To our surprise, we failed to detect any sequences homologous to the $D$. virilis IGS 225 bp repeats in the $D$. americana genome. This family of repeats most likely 


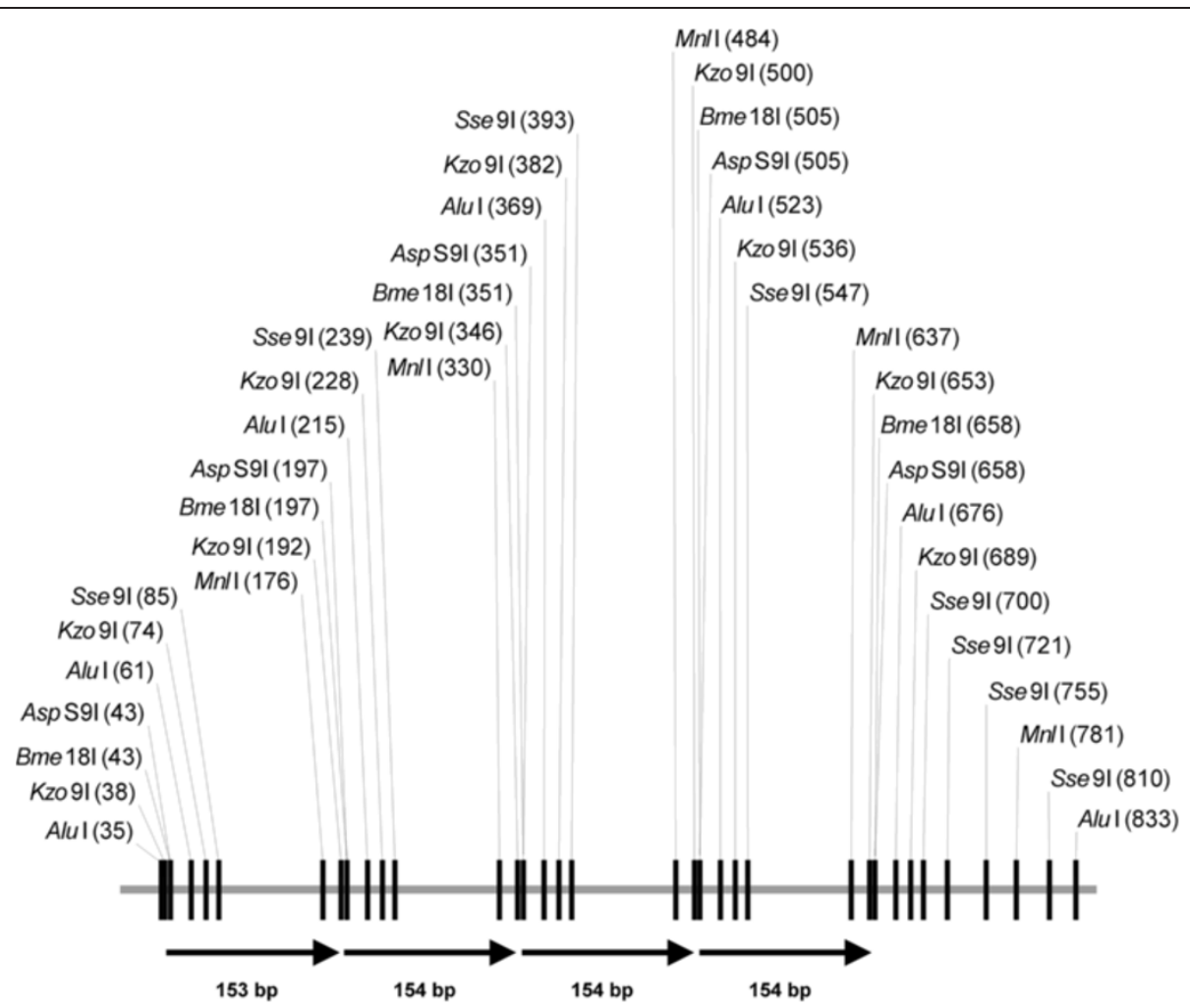

Figure 7 Map of the Helitron-2 fragment that contains tandem repeats. Tandem repeat units are indicated by arrows. The sizes of the units comprising the cluster are given.

appeared in and spread throughout the $D$. virilis genome after the separation of these species. It will be interesting to find out what repeated sequences are present within IGS of D. americana and other species of the group. It is of note that due to its repetitive nature the ribosomal

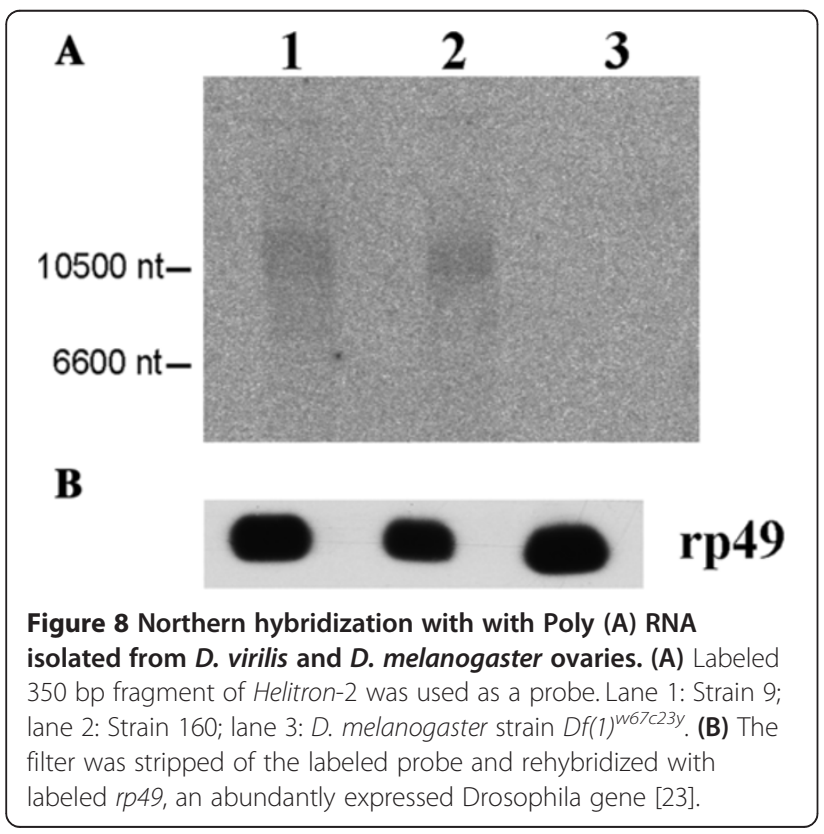

gene region may be difficult to assemble, and this could be the reason why sequences homologous to the IGS 225 bp repeats have not been found so far in D. americana. Unless a scaffold is found with the whole intergenic region and without the repeat, this possibility cannot be altogether discarded. Interestingly, both sequenced genomes of $D$. americana do contain approximately the same number (approximately 150 copies) of 154 bp repeats, showing a high level of similarity (90-95\%) with the consensus sequence of the 154 bp repeats from $D$. virilis, as described above. Because this sequence represents a fragment of the well-known Helitron-2 transposon, it is evident that multiple copies of this mobile element, possibly similarly truncated, are also present in the genome of D. americana. Therefore, invasion and massive amplification of Helitron-2 apparently took place early in the evolution of the virilis phylad group. Similar situation was described in the species of $D$. ananassae subgroup where amplification of another family of 175-200 bp long repeats took place apparently exploring retroposition mechanism [24].

Similarly, our analysis enabled the detection of multiple copies of $172 \mathrm{bp}$ repeats in the genome of $D$. americana. Thus, both investigated strains of $D$. americana contain approximately 180 copies belonging to this family of repeats. We can not say, however, whether the $172 \mathrm{bp}$ 


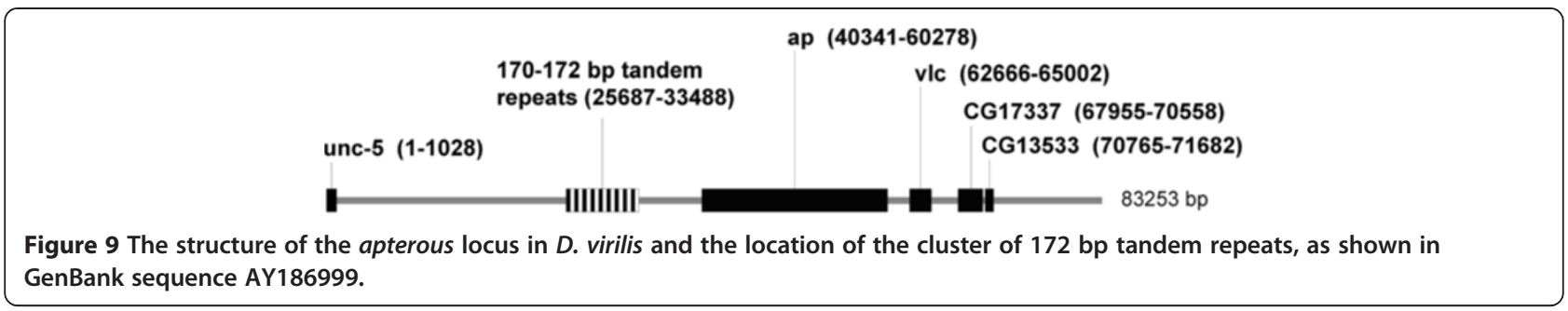

repeats in D. americana are clustered, as is the case in the $D$. virilis apterous region, or scattered throughout the genome. We performed a BLAST search using the $172 \mathrm{bp}$ consensus fragment as a query in other available sequenced Drosophila genomes and did not find any sequences with significant homology to the repeats. Thus, the tandem repeats are apparently specific for certain species of the virilis group of Drosophila. The comparison of the described medium-sized repeats between different species of the virilis group and other related species may be very helpful in understanding the function and origin of these repeated sequences and their possible role in the evolution of close species of Drosophila.

The described method has the potential to learn more about regions containing repeats. The knowledge about long repeats could be used to construct maps of these regions. Even though the digestion method would be laborious, it could potentially help to piece together a genomic sequence of the heterochromatic regions in particularly in species containing large proportion of repeats. Furthermore, the developed method may be used to detect the amplification of various transposable elements ("bursts") by comparison of the restriction patterns of the individual strains and geographical populations of certain species with those of the basic sequenced species strain with partially or completely annotated genome.

\section{Conclusion}

Using in silico digestion in combination with conventional restriction analysis and sequencing of repeated DNA fragments enabled us to isolate and characterize three highly abundant families of medium-sized repeats present in the D. virilis genome. These repeats comprise a significant portion of the $D$. virilis genome and may have important roles in genome function and structural integrity. Interestingly, two of the described families were also abundant in $D$. americana, which belongs to the same phylogenetic group. At the present time, we do not know whether these repeats were formed by unequal crossing-over events, replication slippage or the rolling-circle replication mechanism used in the propagation of Helitron-like transposons. This investigation emphasizes the validity and versatility of in silico digestion method for the detection and analysis of the multiple families of tandem repeats that often escape analysis in the process of genome assembling.
Importantly, the suggested approach may help to shed light on the structure and composition of heterochromatic regions of the sequenced genomes and help to elucidate general trends in heterochromatin evolution.

\section{Methods}

\section{Fly stocks}

In our experiments, we used two strains of $D$. virilis and one D. melanogaster strain. D. virilis strain 160 is an old laboratory strain that carries recessive markers in all autosomes. A derivative of strain 160 was used to determine the genome sequence of $D$. virilis. The second $D$. virilis strain, strain 9, was used for comparison and represents the wild-type strain, caught in 1971 in Batumi, Georgia. In addition, we used the Oregon $\mathrm{R}$ strain of $D$. melanogaster. Flies of all species were reared on standard resin-sugar-yeast-agar medium containing proprionic acid and methylparaben as mold inhibitors.

\section{Isolation and analysis of genomic DNA and mRNA from Drosophila species}

Genomic DNA was isolated from flies using a standard phenol-chloroform extraction technique. Hydrolysis reactions were performed for 2 hours, at optimal temperature, in $20 \mu \mathrm{l}$ of the reaction mixture containing $2 \mu \mathrm{g}$ of DNA, SE-buffers, as recommended by the manufacturer, and $1 \mu \mathrm{l}$ of restriction enzyme. Gel electrophoresis using $8 \%$ agarose gel was conducted in Tris-acetate buffer to separate the DNA fragments. $2 \mu \mathrm{g}$ of hydrolyzed DNA were loaded on agarose gel in each run. After electrophoresis, DNA bands were stained with ethidium bromide and photographed in UV light.

To determine 225 bp HpaII fragments sequences, gel piece with visible 225 bp bands was excised out after electrophoresis. DNA fragments were isolated from gel pieces using QIAEX II Gel Extraction Kit (QIAGEN) and ligated with pUC19 plasmid linearized with SmaI. E. coli XL1-blue competent cells were transformed with obtained ligation mixture. Plasmid DNAs from the grown colonies were isolated using NucleoSpin Plasmid Kit (Macherey-Nagel). The sequences of insertions were determined using ABI Prism 310 Genetic Analyzer (Applied Biosystems).

Total RNA and poly (A)-RNA were extracted from the thoraxes or ovaries of adult flies, as previously described 
[28], and using the TRiZoL reagent (Sigma). The integrity of each RNA preparation was checked on ethidium bromide-stained 1.2\% agarose/Mops-formaldehyde gels.

Radiolabeled probes $\left({ }^{32} \mathrm{P}\right)$ were obtained by random priming of repeat-containing DNA fragments isolated from agarose gels. Five micrograms of poly (A) RNA were loaded in each lane of $1.2 \%$ agarose/Mops-formaldehyde gel. RNA transfer was performed on Hybond XL in $6 \mathrm{xSSC}$ overnight. The membranes were UV crosslinked. Hybridizations were performed overnight at $42^{\circ} \mathrm{C}$ in $50 \%$ formamide, using ultrasensitive hybridization buffer (Ambion).

\section{In situ hybridization with polytene chromosomes and cytological analysis}

Salivary glands were dissected from $D$. virilis third-instar larvae in $45 \%$ acetic acid and squashed according to the described procedures [29]. For in situ hybridization studies, larvae were grown at $18^{\circ} \mathrm{C}$, and a live yeast solution was added to the culture two days before the larvae were analyzed. The DNA probes described above were biotinylated by nick translation using biotin 14-dATP [29]. Chromosomal localizations were made using cytological photographic maps of $D$. virilis [30].

\section{Sequence analysis and diagram plotting}

The distribution diagrams of Drosophila genomic DNA digestion in silico at recognition sites of several restriction endonucleases were constructed according to the previously described techniques [1]. The Tandem Repeat Finder program [31] was used to obtain sequences of repeats that were $40-400 \mathrm{bp}$ in length and arranged in tandem within the Drosophila genomic sequences [32].

\section{Additional file}

Additional file 1: Figure S1. The alignment of $172 \mathrm{bp}$ tandem repeats cluster located close to apterous gene of D. virilis. Deletions are indicated by yellow color, insertions are indicated by blue color, while grey color indicates hexanucleotide which does not have any homology with 172 bp consensus sequence.

\section{Competing interests}

The authors declare that they have no competing interests.

\section{Authors' contributions}

MAC, DAG, VAC, VNT and JET carried out DNA hydrolysis, cloning and sequencing od DNA fragments. NGS and OGZ performed Northern analysis, ESZ performed in situ hybridization experiments. SKD and MBE coordinated the project and wrote the final manuscript. All authors have read and approved the final manuscript.

\section{Funding}

Work was supported by the Russian Foundation for Basic Research, project № 09-04-00643 and 09-04-00660, project from "Genofond dynamics" program, Grant of the Program of Molecular and Cellular Biology RAN to M.E.

\section{Author details}

${ }^{1}$ SibEnzyme Ltd., Ak. Timakova Str. 2/12, Novosibirsk 630117, Russia.

2Engelhardt Institute of Molecular Biology, Vavilov str. 32, Moscow 119991,

Russia. ${ }^{3}$ Institute of Cell Biophysics, Pushchino 142290, Russia.

Received: 30 December 2012 Accepted: 28 October 2013

Published: 9 November 2013

\section{References}

1. Abdurashitov MA, Tomilov VN, Chernukhin VA, Gonchar DA, Degtyarev SK: Mammalian chromosomal DNA digestion with restriction endonucleases in silico. Ovchinnikov bulletin of biotechnology and physical and chemical biology 2006, 2(3):29-38. [Rus] (online english version - http://science.sibenzyme.com/article14_article_27_1.phtml).

2. Chernukhin VA, Abdurashitov MA, Tomilov VN, Gonchar DA, Degtyarev SK: Comparative restriction analysis of rat chromosomal DNA in vitro and in silico. Ovchinnikov bulletin of biotechnology and physical and chemical biology 2006, 2(3):39-46. [Rus] (online english version - http://science.sibenzyme.com/article14_article_28_1.phtml).

3. Abdurashitov MA, Tomilov VN, Chernukhin VA, Degtyarev SK: A physical map of human Alu repeats cleavage by restriction endonucleases. BMC Genomics, 9:305.

4. Palomeque T, Lorite P: Satellite DNA in insects: a review. Heredity 2008, 2008(100):564-73.

5. Plohl M, Luchetti A, Mestrovic N, Mantovani B: Satellite DNAs between selfishness and functionality: structure, genomics and evolution of tandem repeats in centromeric heterochromatin. Gene 2008, 409:72-82.

6. Gall JG, Atherton DD: Satellite DNA sequences in Drosophila virilis. J Mol Biol 1974, 85:633-64

7. Morales-Hojas R, Reis M, Vieira CP, Vieira J: Resolving the phylogenetic relationships and evolutionary history of the Drosophila virilis group using multilocus data. Mol Phylogenet Evol 2011, 60:249-58.

8. Spicer G, Bell C: Molecular phylogeny of the Drosophila virilis species group (Diptera: Drosophilidae) inferred from mitochondrial $12 \mathrm{~S}$ and $16 \mathrm{~S}$ ribosomal RNA genes. Ann Entomol Soc Am 2002, 95:156-61.

9. Evgen'ev MB, Yenikolopov GN, Peunova NI, Ilyin YV: Transsposition of mobile genetic elements in interspecific hybrids of Drosophila. Chromosoma 1982, 85:375-86.

10. Evgen'ev MB, Zelentsova H, Shostak N, Kozitsina M, Barskyi V, Lankenau DH Corces VG: Penelope, a new family of transposable elements and its possible role in hybrid dysgenesis in Drosophila virilis. Proc Natl Acad Sci US A 1997, 94:196-201.

11. Evgen'ev MB, Zelentsova H, Poluectova H, Lyozin GT, Veleikodvorskaja V, Pyatkov Kl, Zhivotovsky LA, Kidwell MG: Mobile elements and chromosomal evolution in the virilis group of Drosophila. Proc Natl Acad Sci U S A 2000, 97:11337-11442.

12. Evgen'ev MB, Arkhipova IR: Penelope-like elements - a new class of retroelements: distribution, function and possible evolutionary significance. Cytogenet Genome Res 2005, 110:510-521.

13. Petrov DA, Schutzman JL, Hartl DL, Lozovskaya ER: Diverse transposable elements are mobilized in hybrid dysgenesis in Drosophila virilis. Proc Natl Acad Sci U S A 1995, 92:8050-8054

14. Morales-Hojas R, Vieira CP, Vieira J: The evolutionary history of the transposable element Penelope in the Drosophila virilis group of species. J Mol Evol 2006, 63:262-273.

15. Heikkinen E, Launonen V, Muller E, Bachmann L: The pvB370 BamHI satellite DNA family of the Drosophila virilis group and its evolutionary relation to mobile dispersed genetic pDv elements. J Mol Evol 1995, 41:604-614.

16. Stage DE, Eickbush TH: Sequence variation within the rRNA gene loci of 12 Drosophila species. Genome Res 2007, 17:1888-1897.

17. Grimaldi G, Fiorentini P, Di Nocera PP: Spacer promoters are orientationdependent activators of pre-rRNA transcription in Drosophila melanogaster. Mol Cell Biol 1990, 10:4667-4677.

18. Mateos M, Markow TA: Ribosomal intergenic spacer (IGS) length variation across the Drosophilinae (Diptera: Drosophilidae). BMC Evol Biol 2005, 5:46.

19. Kohany O, Gentles AJ, Hankus L, Jurka J: Annotation, submission and screening of repetitive elements in repbase: RepbaseSubmitter and censor. BMC Bioinformatics 2006, 7:474.

20. Jurka J, Kapitonov W, Pavlicek A, Klonowski P, Kohany O, Walichiewicz J: Repbase update, a database of eukaryotic repetitive elements. Cytogenet Genome Res 2005, 110:462-467. 
21. Kapitonov W, Jurka J: Helitrons in fruit flies. Repbase Reports 2007, 7:127-32.

22. Yang HP, Barbash DA: Abundant and species-specific Dine-I transposable elements in 12 Drosophila genomes. Genome Biol 2008, 9(2):R39.

23. Connell $P$, Rosbash M: Sequence, structure, and codon preference of the Drosophila ribosomal protein 49 gene. Nucl Acids Res 1984, 12:5495-5514

24. Nozawa M, Kumagai M, Aotsuka T, Tamura K: Unusual evolution of interspersed repeat sequences in the Drosophila ananassae subgroup. Mol Biol Evol 2006, 23:981-987.

25. Cohen B, McGuffin ME, Pfeifle C, Segal D, Cohen SM: Apterous, a gene required for imaginal disc development in Drosophila encodes a member of the LIM family of developmental regulatory proteins. Genes Dev 1992, 6:715-729.

26. Bergman $C M$, Pfeiffer BD, Rincón-Limas DE, Hoskins RA, Gnirke A, Mungall CJ, Wang AM, Kronmiller B, Pacleb J, Park S, Stapleton M, Wan K, George RA, de Jong PJ, Botas J, Rubin GM, Celniker SE: Assessing the impact of comparative genomic sequence data on the functional annotation of the Drosophila genome. Genome Biol 2002, 3(12):RESEARCH0086.

27. Nuno A, Morales-Hojas FR, Reis M, Rocha H, Vieira C, Nolte V, Schlötterer C, Vieira J: Drosophila americana as a model species for comparative studies on the molecular basis of phenotypic variation. Genome Biol Evol 2013, 5:661-679.

28. Schostak N, Pyatkov K, Zelentsova E, Arkhipova I, Shagin D, Shagina I, Mudrik E, Blintsov A, Clark I, Finnegan DJ, Evgen'ev M: Molecular dissection of Penelope transposable element regulatory machinery. Nucleic Acids Res 2008, 6:2522-2529

29. Lim JK: In situ hybridization with biotinylated DNA. Dros Inf Serv 1993 72:73-77.

30. Gubenko IS, Evgen'ev MB: Cytological and linkage maps of Drosophila virilis chromosomes. Genetica 1984, 65:127-139.

31. Benson G: Tandem repeats finder: a program to analyze DNA sequences. Nucleic Acids Res 1999, 27:573-80.

32. Drosophila 12 genomes consortium. Evolution of genes and genomes on the Drosophila phylogeny. Nature 2007, 450:203-218.

doi:10.1186/1471-2164-14-771

Cite this article as: Abdurashitov et al: Medium-sized tandem repeats represent an abundant component of the Drosophila virilis genome. BMC Genomics 2013 14:771.

\section{Submit your next manuscript to BioMed Central and take full advantage of:}

- Convenient online submission

- Thorough peer review

- No space constraints or color figure charges

- Immediate publication on acceptance

- Inclusion in PubMed, CAS, Scopus and Google Scholar

- Research which is freely available for redistribution 